\title{
Massive parallel sequencing in individuals with multiple primary tumours reveals the benefit of re-analysis
}

\author{
Karin Wallander ${ }^{1,2^{*}}$, Håkan Thonberg $^{1,2}$ (D), Daniel Nilsson ${ }^{1,2+}$ (D) and Emma Tham ${ }^{1,2+}$ (D)
}

\begin{abstract}
Multiple primary cancers, defined as three or more primary tumours, are rare, and there are few genetic studies concerning them. There is a need for increased knowledge on the heritability of multiple primary cancers and genotype-phenotype correlations. We have performed whole-genome/exome sequencing (WGS/WES) in ten individuals with three or more primary tumours, with no previous findings on standard clinical genetic investigations. In one individual with a clinical diagnosis of MEN1, a likely pathogenic cryptic splice site variant was detected in the MEN1 gene. The variant (c.654C > A) is synonymous but we showed in a cDNA analysis that it affects splicing and leads to a frameshift, with the theoretical new amino acid sequence p.(Gly219Glufs*13). In one individual with metachronous colorectal cancers, ovarian cancer, endometrial cancer and chronic lymphocytic leukaemia, we found a likely pathogenic variant in the $M L H 1$ gene (c.27G > A), and two risk factor variants in the genes CHEK2 and HOXB13. The MLH1 variant is synonymous but has previously been shown to be associated to constitutional low-grade hypermethylation of the MLH1 promoter, and segregates with disease in families with colorectal and endometrial cancer. No pathogenic single nucleotide or structural variants were detected in the remaining eight individuals in the study. The pathogenic variants found by WGS/WES were in genes already sequenced by Sanger sequencing and WES in the clinic, without any findings. We conclude that, in individuals with an unequivocal clinical diagnosis of a specific hereditary cancer syndrome, where standard clinical testing failed to detect a causative variant, re-analysis may lead to a diagnosis.
\end{abstract}

Keywords: Hereditary cancer, Re-analysis, WGS, WES, Multiple primary, MEN1, MLH1

\section{Introduction}

Globally, cancer is the second most common killer and accounts for $16 \%$ of all deaths [1]. In Sweden, the most common types of cancer are prostate cancer, breast cancer, and non-melanoma skin cancer [2]. The risk of a second cancer in individuals who have already had one is higher than in the general population [3]. Multiple primary tumours are defined by the Surveillance

\footnotetext{
* Correspondence: karin.wallander@ki.se

${ }^{\dagger}$ Nilsson Daniel and Tham Emma contributed equally to this work. 'Department of Molecular Medicine and Surgery, Karolinska Institutet, Stockholm, Sweden

2Department of Clinical Genetics, Karolinska University Hospital, Stockholm, Sweden
}

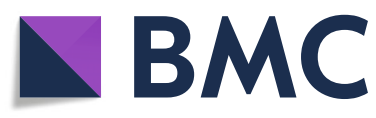

(c) The Author(s). 2021, corrected publication 2021. Open Access This article is licensed under a Creative Commons Attribution 4.0 International License, which permits use, sharing, adaptation, distribution and reproduction in any medium or format, as long as you give appropriate credit to the original author(s) and the source, provide a link to the Creative Commons licence, and indicate if changes were made. The images or other third party material in this article are included in the article's Creative Commons licence, unless indicated otherwise in a credit line to the material. If material is not included in the article's Creative Commons licence and your intended use is not permitted by statutory regulation or exceeds the permitted use, you will need to obtain permission directly from the copyright holder. To view a copy of this licence, visit http://creativecommons.org/ licenses/by/4.0/. The Creative Commons Public Domain Dedication waiver (http://creativecommons.org/publicdomain/zero/1. 0/) applies to the data made available in this article, unless otherwise stated in a credit line to the data. national Association of Cancer Registries (IACR) as two or more histologically distinct tumours, not caused by metastasis, recurrence, or local spread, and diagnosed in the same individual $[4,5] .0 .1-0.2 \%$ of all patients with cancer are estimated to have at least three different primary malignancies [6,7], but reports on cohorts with three or more primary tumours are rare. In Sweden, 3\% of all tumours registered between 2014 and 2018 were the third tumour discovered in that individual. It is important to note, however, that those are not necessarily primary tumours, since the numbers include recurrences 
and only synchronous tumours in the same organ had been excluded [8].

Multiple primary tumours can be caused by genetic predisposition, common exposure of carcinogenic agents, an underlying developmental abnormality, or chance. There are some already defined hereditary cancer syndromes that are associated with an increased risk of multiple primary tumours, such as Lynch syndrome and Li-Fraumeni syndrome [9]. However, we do not yet have full knowledge on the association between a specific pathogenic gene variant and all potential cancers that it can give rise to. An example of this is the CHEK2 gene, wherein there are pathogenic variants that give rise to an increased risk for breast cancer but the extent of risks for other cancers is under debate [10, 11].

Knowledge on the genetic background of hereditary cancer is crucial for the treatment and follow-up of patients and their families. At clinical genetics departments, we offer genetic screening based on the combination of cancer types within the family. Usually, testing is done using a predefined cancer gene panel. For individuals with three or more primary tumours who do not fulfil clinical criteria, or in whom no causative variants are detected in clinical testing, there is no consensus concerning additional genetic testing. It has been suggested that whole-genome/exome sequencing (WGS/ WES) should be considered for this patient cohort [12], but the clinical benefits of WGS/WES compared to standard clinical gene panels are arguable. Therefore, we performed WGS/WES in ten individuals with three or more primary tumours, to investigate if these techniques could identify novel germline genetic variants and thus provide additional clinical utility.

\section{Materials and methods}

Participating families were recruited from the Department of Clinical Genetics at Karolinska University Hospital, Stockholm, Sweden, between 2013 and 2015. The inclusion criteria were three or more primary tumours with the first tumour before the age of 60 years (or four or more primary tumours at any age) in one individual in the family, with no obvious etiological cause. All participants had been clinically investigated for known cancer syndromes, either by targeted genetic analyses or by medical history.

Blood samples were collected in EDTA tubes and DNA was extracted according to standard procedures using Qiagen DNA extraction kit (QIAsymphony, DSP DNA Midi Kit, Hilden, Germany). WGS/WES was performed on the Illumina 2500 platform (San Diego, CA, USA) at the Department of Clinical Genetics and the Science for Life Laboratory, Solna Sweden, according to clinical procedures [13, 14] in 2015 and 2016. The first four included samples (from participant F, G, H and I) were analysed using WES, and for the other six samples (from participant A, B, C, D, E, and J) WGS was used. For WES, an average of $175 \mathrm{M}$ reads were generated, for a $156 \mathrm{x}$ average coverage $(98.1 \%$ of bases to $20 \mathrm{x})$ in OMIM morbid genes $[15,16]$. For WGS, an average of $861 \mathrm{M}$ reads were generated, for a 37.0x coverage $(99.0 \%$ of bases to 20x) in OMIM morbid genes. See Supplementary Table S1 for individual values. Read alignment, variant calling and variant annotation was performed with MIP v9.0.2 (https://github.com/Clinical-Genomics/ MIP) and analysis and interpretation in Scout v4.29 (https://github.com/Clinical-Genomics/scout).

Three separate variant selection approaches were used, as can be seen in Supplementary Fig. S1. Fist, a custom filtering of all variants in the VCF file was applied. An in silico cancer gene list of 302 genes had been applied; a merge between a list of known somatic driver genes defined by Vogelstein et al. [17] and a local list of hereditary cancer associated genes, see Supplemental Table S2A. Only genetic variants with a maximal minor allele frequency (MMAF) lower than $0.1 \%$ in ExAC [18] and with an allele fraction (alternate/(reference + alternate) allele ratio) above $30 \%$ were considered. All variants known to be pathogenic/likely pathogenic for a welldefined hereditary cancer syndrome according to ClinVar [13] [19], were included and variants reported to be benign by more than one source were excluded. In addition, exonic or splice variants were included, and synonymous variants were only included if they had a predicted effect on splicing by SpliceSiteFinder-like, MaxEntScan, GeneSplicer, and NNSPLICE [20]. The predictions on protein effect were extracted from Align GVGD [21] [22], SIFT [23], PolyPhen2 [24], and Mutation Taster [25]. The Alamut software (Alamut Visual, Interactive Biosoftware, Rouen, France) was used to access and visualise the predictions. It was also noted if the variant had been reported as a somatic cancer mutation in the database cBioPortal [26]. The ACMG (American College of Medical Genetics and Genomics) classification system was followed for pathogenicity annotation for all filtered variants [27]. We also checked if there were specific adjustments to the ACMG criteria stipulated by the Clinical Genome Resource ClinGen for the genes investigated [28]. Heterozygous variants judged to be pathogenic according to the ACMG criteria, but not considered disease-causing in the participant, were classified as pathogenic incidental findings (for autosomal recessive conditions) or risk factors.

Secondly, the variants were ranked using the software Genmod (https://github.com/moonso/genmod) and visualized in Scout [29]. An in silico gene list related to the human phenotype ontology term HP:0002664 Neoplasm (accessed on 11-25-2019) and a curated gene list from the Department of Clinical Genetics 
(Supplementary Table S2B and S2C) were used. Scout allows variant triage by display of ranked variant lists with relevant annotation information, such as population frequency, local variant frequency, previous clinical classification in e.g. ClinVar, conceptual functional annotation by VEP and common variant effect prediction scores including CADD, SIFT and PolyPhen [30] [23] [24]. All variants were examined in two analyses, one predicting a dominant disease and the other searching for variants associated to a recessive disease (homozygous and compound heterozygous variants). Genetic sequence variants were excluded if they were classified as benign/likely benign by ClinVar by multiple sources [19], if they occurred in a non-coding, non-splice-site region, if they were reported to have occurred more than three times in another cohort (among 4755 individuals tested with WGS/WES locally) or had a MMAF $>0.1 \%$ globally [18]. Variants in a gene known to cause a specific syndrome were considered less relevant if the individual harbouring that variant had no clinical features of that syndrome. The selected variants were ranked according to the same criteria as the variants from the initial cancer gene lists, and classified using the ACMG criteria.

Thirdly, we created a shortlist of genes which were specifically interesting in each participant according to their cancer diagnoses and their family history (see Supplementary Table S3). We checked all variants including non-coding/synonymous variants in those genes. Sequence variants were excluded if they were classified as benign/likely benign by ClinVar by multiple sources [19], or had a MMAF $>0.1 \%$ globally [18]. Non-coding variants were checked for occurrence in the Blueprint genetic list of non-coding variants included in their genetic hereditary cancer screening test [31].

Finally, we used Scout to find structural variants (SVs) in the data from the six individuals who had had a WGS analysis. Scout uses four SV callers: Delly, Manta, CNVator and TIDDIT [32-35] and filters against a reference database with 1000 Swedes [36] and our local database of 4755 individuals. The in silico cancer gene list from the Department of Clinical Genetics, Karolinska, (Supplementary Table S2C) was used and all SVs were examined. Variants were excluded if they occurred in more than five persons in the local database, or were likely artefacts (either occurring in repetitive genome areas and/ or large copy number variants called by primarily discordant read pair focused callers (Manta, Delly) [33] [32] and not supported by read coverage (as e.g. CNVnator) [34]). As with single nucleotide variants, variants that were not predicted to modify expressed gene products (e.g. intronic duplications or inversions with intergenic breakpoints) were dismissed from further triage.
For expression analysis of the MEN1 gene, blood was collected from participant $\mathrm{E}$ in a PAXgene ${ }^{\circ}$ Blood RNA Tube (PreAnalytiX GmbH, Switzerland) and RNA was isolated with PAXgene Blood RNA Kit accordingly to the manufacture's instructions (PreAnalytiX GmbH, Switzerland). Subsequently, cDNA was prepared with reverse transcriptase SuperScript ${ }^{\circ}$ VILO $^{\mathrm{ma}}$ MasterMix (Thermo Fisher Scientific, MA USA), and sequenced with Sanger sequencing using specific primers; forward 5' -CGTGAGCTGGTGAAGAAGGT-3', and reverse, $5^{\prime}$ GTCCCAGGTCATAGAGCAGC-3'. PCR was performed with AmpliTaq Gold (ThermoScientific, US) with the following cycle-program: $96^{\circ} \mathrm{C} 10 \mathrm{~min},\left(96^{\circ} \mathrm{C}\right.$ $\left.30 \mathrm{~s}, 62^{\circ} \mathrm{C} 30 \mathrm{~s}, 72^{\circ} \mathrm{C} 40 \mathrm{~s}\right) \times 35,72{ }^{\circ} \mathrm{C} 8 \mathrm{~min}$.

\section{Results}

Ten individuals with three or more primary tumours were included in the study, eight women and two men. The participants' phenotypes are listed in Table 1.

Two participants, A and E, fulfilled the clinical criteria for specific hereditary cancer syndromes. Participant A had metachronous cancers in the colon (with defect mismatch repair), ovaries, and uterus, with her first cancer at the age of 30 . Her father had colorectal cancer at the age of 76. This is highly suggestive of Lynch syndrome or Lynch-like syndrome. Participant E fulfilled the clinical criteria for MEN1 syndrome (multiple endocrine neoplasia type 1) [37], with parathyroid hyperplasia (at 27 years of age), prolactin and GH producing pituitary adenoma, and multiple neuroendocrine tumours in the pancreas, all of them less than one $\mathrm{cm}$. She also had a malignant melanoma of $1.4 \mathrm{~mm}$ at the age of 33 . Her mother was operated for primary hyperparathyroidism at 47 years of age. She also had a prolactin-producing microadenoma of the pituitary and multiple lipomas.

Four participants had possible hereditary cancer syndromes, based on age of cancer onset or occurrence of multiple tumours known to be associated to the same syndrome. Participant B had a suspected familial colorectal cancer syndrome. Participant $\mathrm{C}$ and $\mathrm{H}$ both had suspected familial breast and ovarian cancer syndromes. Participant $\mathrm{C}$ had a mother with ovarian cancer at the age of 57, a sister with breast cancer at the age of 62, and a father with prostate cancer at the age of 61 , and participant $\mathrm{H}$ had a brother with testicular cancer at the age of 36. The clinical criteria for familial malignant melanoma $[38,39]$ were met in the family of participant $\mathrm{D}$, who had malignant melanoma at 59 years of age, and a brother with malignant melanoma at 40 years of age.

The remaining four participants did not fulfil any hereditary cancer syndrome criteria. In the family of participant I, both the brother and the father had prostate cancer at 65 and 80 years of age, respectively, suggestive of familial prostate cancer. Participant J had early onset 


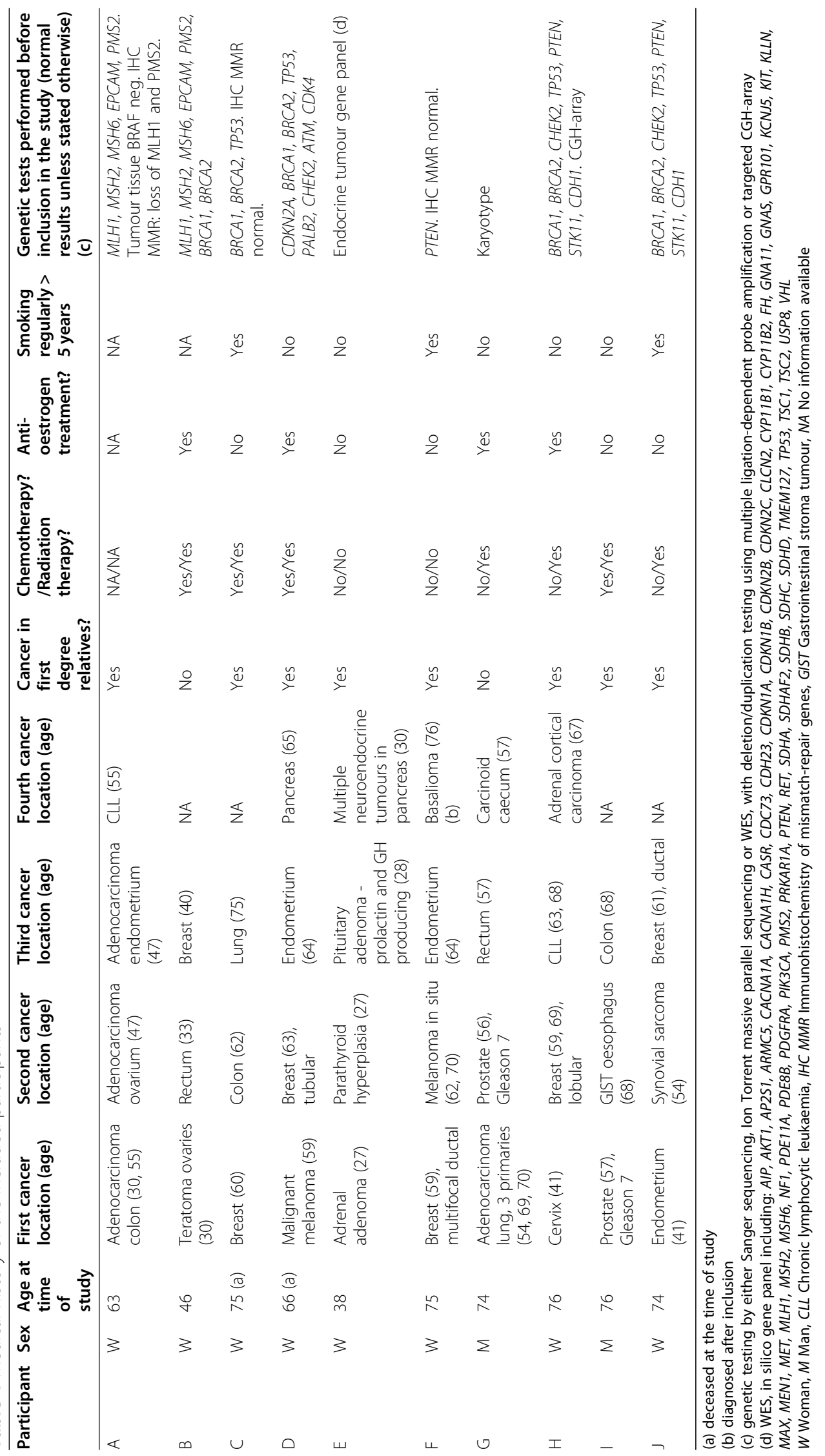


uterine cancer, but unfortunately, no tissue biopsy was available and no microsatellite instability or immunohistochemistry analysis for mismatch-repair proteins could be performed. Her family history was not suggestive of Lynch syndrome.

In the ten participants, a total of 32 potential cancer syndrome candidate single nucleotide variants were identified from WGS/WES, see Table 2. All of the variants occurred in a heterozygous state and a majority were missense. No structural variants relevant for cancer were detected.

A clinically highly relevant synonymous variant was found in the MEN1 gene in participant E, with a clinical diagnosis of MEN1. Her DNA had been sequenced twice via the Department of Clinical Genetics at Karolinska University Hospital (using specific Sanger sequencing and MLPA of the MEN1 gene in 2009, and wholeexome sequencing and an in silico gene list for endocrine tumours in 2015), with no pathogenic variant found (Table 1). The synonymous c.654C > A MEN1 variant was detected in the clinical testing, but it was classified as likely benign and not mentioned in the report. The variant had been reported as a likely benign variant in ClinVar (variation ID 748511, accessed 07-032021), by a single submitter and without further details [19]. However, in the present analysis, the variant was predicted by the Alamut software to potentially strengthen a cryptic splice site (SpliceSiteFinder-like from 71.5 to 80.4 , MaxEntScan, 5 to 6.3 , GeneSplicer, 4.3 to 7.6 , and NNSPLICE 0 to 0.8 ) [20], leading to a deletion of 14 nucleotides in exon 3 and a frameshift in the RNA sequence (Fig. 1). The variant is not present in a reference database (gnomAD v2.1.1) [41]. The variant was therefore investigated further by cDNA sequencing. This revealed that the individual had a frameshift, as predicted, and the conceptual new amino acid sequence p.(Gly219Glufs"13). Based on the information from the functional analysis, the variant fulfilled the specific ACMG criteria PS3, PM2 and PP4 and it was classified as likely pathogenic; with a spliceogenic loss-of-function effect [27]. The family members had been tested for MEN1 biochemically before this study, and the mother was diagnosed with a primary hyperparathyroidism and underwent surgery at the age of 47. The MEN1 variant was inherited from the affected mother and absent from the healthy father, and the 38-year-old healthy sister.

The sequence reads from cDNA and gDNA from participant $\mathrm{E}$, followed by a depiction of the activation of a cryptic splice site 14 nucleotides upstream of the normal splice site at the 3 ' end of exon 3 . The novel splice site leads to a loss of 14 nucleotides and a frameshift on the mRNA level.

Using the approach of targeting genes associated to the participants' specific cancer diagnoses yielded one more likely pathogenic variant: the $M L H 1$ variant in participant A. The variant had been detected by Sanger sequencing performed at the Department of Clinical Genetics, Karolinska University Hospital, in 2012, but as it was synonymous and another nucleotide change at the same position was common in gnomAD, it was considered likely benign. The variant was not predicted to affect splicing by the Alamut software. When the variant was found in our study, though, it had been reported in ClinVar as likely pathogenic by two sources (variation ID 186982, accessed 07-03-2021) [19] [41]. The variant fulfilled the specific ACMG criteria PS4, PM2 and PP4 [27]. Therefore, it was considered likely pathogenic. Immunohistochemistry of participants A's colon cancer biopsy demonstrated loss of protein expression of MLH1 and PMS2, indicative of $M L H 1$ loss of function, and no $B R A F$ V600 mutation was found in tumour DNA.

Two known pathogenic moderate cancer risk variants were also found in participant $\mathrm{A}$; in the genes CHEK2 and HOXB13 (Table 2). In the rest of the participants, no pathogenic variant was found.

Three individuals were heterozygous carriers of variants that are known to cause autosomal recessive conditions if found in a homozygous or compound heterozygous state. These variants, in the genes $R M R P$, $T Y R$, and $P N P$, were classified as incidental findings.

Participant G carried a rare CTNNB1 variant, p.(Met107Val). In order to determine if this variant affected the protein expression of catenin $B$, immunohistochemistry analysis was performed on tumour tissue from participant G at the Department of Clinical Pathology, Karolinska University Hospital, Sweden. A normal pattern of catenin B staining in the cell membranes was found in tumour tissue from the prostate, rectum and initial lung tumour, with a weaker signal in the neuroendocrine tumour. The two later lung tumours demonstrated both membranous and cytoplasmic staining with some nuclear staining in the adenocarcinoma of the right lung.

No variants remained after filtering for participants D and I.

\section{Discussion}

Most publications concerning predisposition to multiple primary tumours include predominantly individuals with two primary malignancies and reports on cohorts with three or more are rare. Multiple primary tumours may be caused by a monogenic predisposition, but may also be due to multifactorial risk factors. There is no clinical consensus on which genetic investigation is appropriate for patients with three or more primary tumours. It is clear that more knowledge is needed before clinical criteria for testing in this patient cohort can be stipulated. We chose to only include individuals with at least three 
Table 2 All selected variants

\begin{tabular}{|c|c|c|c|c|c|c|c|c|}
\hline Participant & $\begin{array}{l}\text { Gene } \\
\text { symbol }\end{array}$ & Refseq & $\begin{array}{l}\text { Nucleotide } \\
\text { substitution }\end{array}$ & $\begin{array}{l}\text { Predicted protein } \\
\text { alteration }\end{array}$ & $\begin{array}{l}\text { VAF } \\
\text { (a) }\end{array}$ & $\begin{array}{l}\text { cBioportal } \\
\text { (b) }\end{array}$ & gnomAD & Variant interpretation \\
\hline A & CHEK2 & $\begin{array}{l}\mathrm{NM}_{-} \\
001005735.1\end{array}$ & 1229delC (1100delC) & Thr410Metfs*15 & 0.4 & $A b$ & 0.008717 & Risk factor \\
\hline A & SDHA & NM_004168.2 & $1724 C>T$ & Ala575Val & 0.4 & $\mathrm{BC}$ & 0.0001386 & VUS \\
\hline A & $A B C B 11$ & NM_003742 & $1724 G>A$ & Arg575Gln & 0.4 & $\mathrm{Bb}$ & 0.0003315 & VUS \\
\hline A & HOXB13 & NM_006361 & $251 G>A$ & Gly84Glu & 0.5 & $A c$ & 0.007618 & Risk factor \\
\hline A & MLH1 & NM_000249 & $27 G>A$ & Arg9= & 0.5 & $\mathrm{Ba}$ & 0 & Likely pathogenic \\
\hline B & CHEK2 & $\begin{array}{l}\mathrm{NM}_{-} \\
001005735.1\end{array}$ & $670 C>T$ & Arg224Cys & 0.7 & $\mathrm{Ab}$ & 0.0005227 & VUS \\
\hline B & KLLN & $\begin{array}{l}\mathrm{NM}_{-} \\
001126049.1\end{array}$ & $422 \mathrm{G}>\mathrm{A}$ & Thr141Met & 0.6 & C & 0 & VUS \\
\hline B & REST & NM_005612 & $968 \mathrm{~T}>\mathrm{G}$ & Met323Arg & 0.5 & Ac & 0 & VUS \\
\hline B & TRIM28 & NM_005762 & $2381 C>T$ & Thr794Met & 0.5 & $A b$ & 0.0001986 & VUS \\
\hline B & LZTR1 (c) & NM_006767 & 1866_1867delTC & Pro623ThrfsTer45 & 0.5 & $\mathrm{Bb}$ & 0 & VUS \\
\hline C & BAP1 & NM_004656.3 & $944 \mathrm{~A}>C$ & Glu315Ala & 0.4 & $\mathrm{Bb}$ & 0.0003263 & VUS \\
\hline C & FBXW7 & $\begin{array}{l}\mathrm{NM}_{-} \\
001013415\end{array}$ & $248 G>A$ & Arg83Lys & 0.5 & $\mathrm{BC}$ & 0.0005178 & VUS \\
\hline C & $R M R P(\mathrm{c})$ & NR_003051 & $71 A>G$ & NA & 0.4 & C & 0.008687 & $\begin{array}{l}\text { Pathogenic incidental } \\
\text { finding }\end{array}$ \\
\hline C & $S M O$ & NM_005631.4 & $517 C>T$ & Arg173Cys & 0.5 & $\mathrm{Bb}$ & 0.0007454 & VUS \\
\hline E & $A R I D 1 B$ & NM_020732.3 & $4727 C>T$ & Pro1576Leu & 0.4 & $A c$ & 0.00003098 & VUS \\
\hline E & MLHI & NM_000249.3 & $41 C>T$ & Thr14lle & 0.7 & $\mathrm{BC}$ & 0.000008792 & VUS \\
\hline E & $S S X 1$ & NM_005635 & 293dupA & Met99AspfsTer24 & 0.6 & $\mathrm{BC}$ & 0.0002149 & VUS \\
\hline$E$ & FAT4 & NM_024582 & $7751 C>A$ & Ser2584Tyr & 0.5 & $\mathrm{BC}$ & 0.00005789 & VUS \\
\hline E & TOP2A & NM_001067 & $154 \mathrm{G}>\mathrm{A}$ & Gly52Ser & 0.4 & $\mathrm{BC}$ & 0 & VUS \\
\hline E & MEN1 & NM_000244 & $654 C>A$ & Ala213= & 0.6 & $\mathrm{Bc}$ & 0 & Likely pathogenic \\
\hline $\mathrm{F}$ & $\operatorname{TYR}(\mathrm{c})$ & NM_000372.4 & $1147 G>A$ & Asp383Asn & 0.6 & $A b$ & 0.0001784 & $\begin{array}{l}\text { Pathogenic incidental } \\
\text { finding }\end{array}$ \\
\hline G & ERCC3 (c) & NM_000122.1 & $1204 G>A$ & Gly402Ser & 0.5 & $\mathrm{BC}$ & 0.00004006 & VUS \\
\hline G & CTNNB1 & $\begin{array}{l}\mathrm{NM}_{-} \\
001098210.1\end{array}$ & $319 A>G$ & Met107Val & 0.5 & $A C$ & 0 & VUS \\
\hline G & TCF3 & NM_003200 & $689 C>G$ & Pro230Arg & 0.5 & $\mathrm{BC}$ & 0.00001430 & VUS \\
\hline $\mathrm{H}$ & RNF6 & NM_005977 & $1780 C>A$ & Leu594lle & 0.4 & $\mathrm{BC}$ & 0 & VUS \\
\hline H & RNF6 & NM_005977 & $895 G>C$ & Glu299GIn & 0.3 & $B C$ & 0.000008792 & VUS \\
\hline H & ARHGAP26 & NM_015071 & $619 C>G$ & Leu207Val & 0.5 & $B C$ & 0.00001760 & VUS \\
\hline H & $X R C C 3$ & NM_005432 & $172 C>T$ & Arg58Trp & 0.5 & $A C$ & 0.00006208 & VUS \\
\hline H & TSC2 & NM_000548 & $1244 C>T$ & Ala415Val & 0.5 & $A C$ & 0.0001866 & VUS \\
\hline J & TCF3 & NM_003200 & c. $1213 C>T$ & p.Arg405Cys & 0.5 & $A c$ & 0.00008796 & VUS \\
\hline J & $P N P(\mathrm{c})$ & NM_000270 & c.701G >C & p.Arg234Pro & 0.6 & $C$ & 0.0001471 & $\begin{array}{l}\text { Pathogenic incidental } \\
\text { finding }\end{array}$ \\
\hline J & ARHGAP26 & NM_015071 & c. $1559 A>G$ & p.GIn520Arg & 0.5 & C & 0.00006175 & VUS \\
\hline
\end{tabular}

VUS Variant of unknown significance

(a) VAF; variant allele fraction (alternate/(reference + alternate))

(b) cBioPortal [26]:

A: Same variant

B: Other similar variant in proximity

C: No similar variant in proximity

a: Same tumour types

b: Overlapping tumour types

c: Other tumour types

(c) Gene associated to potentially cancer-associated syndrome described in OMIM (Online Inheritance in Man) [40] with a recessive inheritance pattern, including those with both recessive and dominant inheritance patterns 


\section{exon junction 3-4}
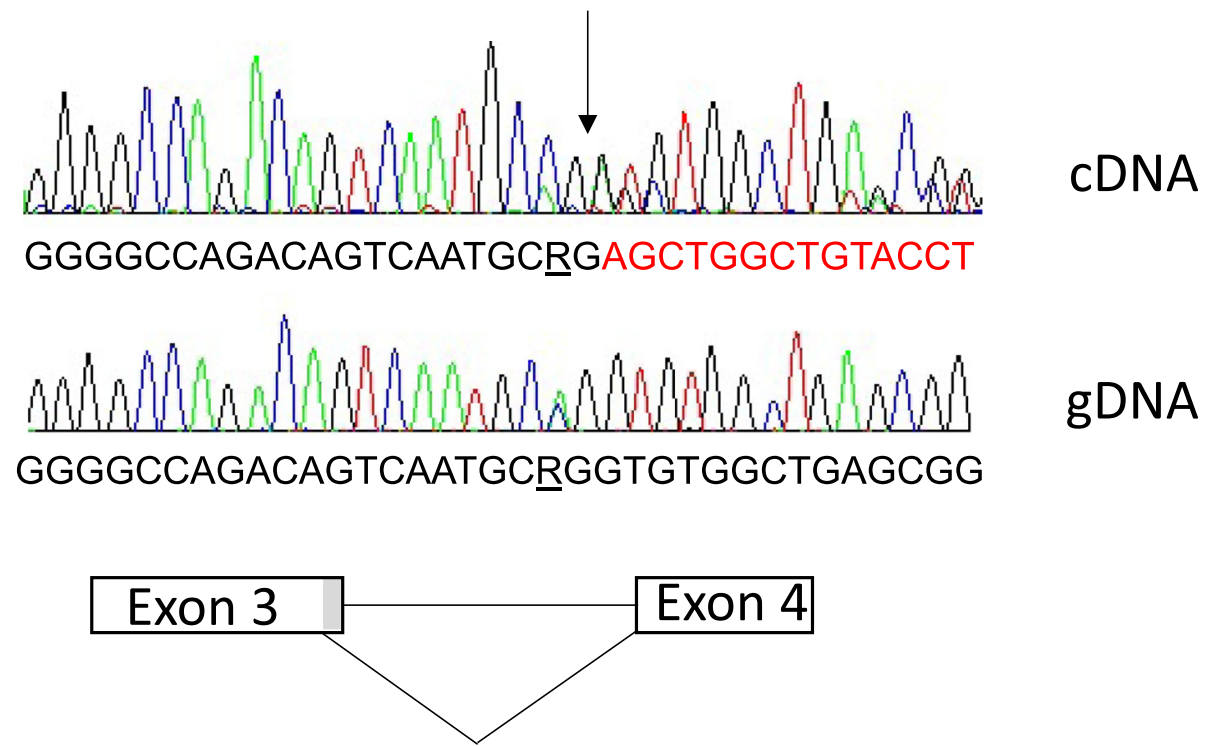

\section{cryptic splice site activated \\ p.(Gly219Glufs*13)}

Fig. 1 Effect of the MEN1 variant on splicing

different primary tumours in this study in order to select a rare group (incidence $0.1-0.2 \%$ [6] [7]) and to minimize the risk of only including secondary therapyrelated cancers.

The WGS analysis led to the detection of a coding variant in the MEN1 gene in participant E. Multiple endocrine neoplasia syndrome 1 (MIM \#131100) is characterised by a predisposition to many different endocrine neoplasms, in particular of the parathyroid, pancreas, and anterior pituitary gland. It is inherited in an autosomal dominant fashion and caused by pathogenic, mostly truncating, variants in the tumour suppressor gene MEN1 [37, 42]. 46 of 356 known pathogenic/likely pathogenic variants in $M E N 1$ are splice site variants [19, 43]. Participant $E$ had an established clinical diagnosis of MEN1, with three major manifestations as well as an adrenal gland adenoma. Although the variant had been reported as likely benign once in ClinVar (variation ID 748511, accessed 07-03-2021), it was not present in a reference population and was predicted to affect splicing. Therefore, we further investigated the functional effects of the variant. cDNA sequencing confirmed that the variant causes a frameshift on the RNA level and can thus be classified as class 4/likely pathogenic by ACMG criteria. The pathogenicity is further supported by the fact that the mother of participant $\mathrm{E}$, who also had MEN1 clinically, is a carrier of the variant and the healthy sister and father are not. Although we detected this variant by WGS, it was not the WGS per se that enabled the finding, but rather a thorough investigation of the coding sequence of the MEN1 gene. There are suggestions on adjustments of the ACMG criteria for $M E N 1$ variant annotations, with more emphasis on the clinical picture and family history [44]. This further underlines the need for evaluating the variant in light of the participant's syndrome, and not excluding variants with a benign reputation. Indeed, in $10-30 \%$ of patients with a clinical diagnosis of MEN1 syndrome, no pathogenic MEN1 variant is found, and it has been suggested that re-analysis of these patients might be beneficial [45]. In a recent study by Backman et al., three out of 14 patients with MEN1 syndrome, but no pathogenic MEN1 variant found on clinical genetic testing, actually did carry a pathogenic variant when re-analysed [46]. The other likely pathogenic variant found in this study, the c.27G > A $M L H 1$ variant in participant A, was found by the approach of including all rare synonymous variants in candidate genes, regardless of their effect on splicing. The variant found in this study has been found in a study performed by Ward et al., in two individuals: one with early onset (22 years), the other with multiple colorectal cancers (50, 52 and 54 years), both with MLH1 loss on immunohistochemistry analysis and low-level constitutional hypermethylation of the $M L H 1$ promoter. Two family members with colorectal cancer at older age (49 and 61 years) did not carry the variant, but the 
microsatellite status of their tumours was unknown [47]. The same variant has also been described by Leclerc et al., in a family fulfilling the Amsterdam I criteria. Low-level constitutional MLH1 hypermethylation and the c.27G > A variant segregated with disease within the family. It was also found on a different haplotype in an individual with colorectal and endometrial cancer from another family [48]. In the ClinVar entry for the present variant, Ambry Genetics state that low-level MLH1 promoter methylation and the c.27G > A variant segregate with Lynch syndrome in their internal data material and that their RNA studies have shown the variant does not cause abnormal splicing (variation ID 186982, accessed 07-03-2021) [19]. The variant was classified as likely pathogenic in our study. It is again clear that reevaluation and not the WGS per se was the most important aspect of this discovery. Of note, participant A had in total five different primary tumours: two colonic, one endometrial, one ovarian, and a chronic lymphocytic leukaemia.

Thus, if a patient fulfils clinical criteria of a specific cancer syndrome it is worthwhile to re-examine the clinical WGS/WES data for rare synonymous/splice site variants. The benefits of re-analysis of genomic data have been previously shown, for instance in cohorts of children with developmental disabilities or congenital syndromes $[49,50]$. Patients with a high suspicion of a specific hereditary cancer syndrome will likely also benefit from periodic re-analysis. Stafford et al. emphasize the importance of re-analysis of genes involved in known tumorigenesis molecular pathways in ovarian cancer patients [51] but further reports on WGS/WES re-analysis in cancer cohorts are so far rare. There is a need for more knowledge on the advantages and disadvantages of this approach in the clinical setting. Similarly, there could potentially be variants in this study that end up being classified as pathogenic in the future.

Also, in participant $\mathrm{A}$, two variants classified as risk factors were discovered in CHEK2 and HOXB13. CHEK2 is considered to harbour moderate-penetrance cancersusceptibility genetic variants, and the most well-studied is the variant found in our study. It is commonly called 1100delC, p.(Thr367fs), but with the most recent nomenclature named c.1229delC, p.(Thr410fs). The truncating c.1229delC variant is known to be a moderaterisk variant for hereditary breast cancer, with the relative risk estimated to be 3.0, and a cumulative risk for breast cancer by 80 years of age of 32\% [52]. In clinical practice in Sweden today, female carriers of truncating CHEK2 variants, who also have a first degree relative with breast cancer, are offered annual mammograms at ages 40 to 60 [53] [54]. Guidelines differ internationally though, and the NCCN (National Comprehensive Cancer Network) recommend all female CHEK2 truncating variant carriers, regardless of family history, to undergo annual screening, in some cases with breast MRI and contrast, starting at or before 40 years of age [55]. In the United Kingdom, annual mammography at ages 40 to 50 is recommended for all carriers of truncating variants in the CHEK2 gene, regardless of family history [56]. Truncating CHEK2 variants have also been shown to confer a slightly increased risk for gastric cancer, kidney cancer, sarcoma, prostate cancer [10], and colorectal cancer [11], with the relative risk for the latter estimated to be 1.88 [52]. These risks are too low to be used to recommend surveillance in the clinic. Participant A's cancers are all likely caused by the Lynch syndrome variant; the CHEK2 variant is less likely to impact on her phenotype. Since participant A did not have any known relative with breast cancer, she and her close relatives were not recommended breast cancer surveillance and no predictive CHEK2 testing could be performed in her family.

Missense variants in the CHEK2 gene may confer a small increase in the risk of cancer, but robust data is only available for the missense variant c.599 T > C, which leads to an estimated relative risk of breast cancer of 1.58 and of colorectal cancer of 1.56 [52]. Individual assessment is recommended for non-truncating CHEK2 variants [55]. The CHEK2 variant c. $670 \mathrm{C}>\mathrm{T}$ (historically, and more commonly known as NM_007194.4: c.541C > T, p. (Arg181Cys)) found in participant B, has been classified as of unknown significance by multiple submissions to ClinVar (variation ID 5597, 24-03-2021) [19]. It is situated in the forkhead-associated (FHA) domain [57], just as the c.599 $\mathrm{T}>\mathrm{C}$ variant. Disturbances of this region can obstruct the binding of CHEK2 to BRCA1 [58]. The c.670C $>\mathrm{T}$ variant has been reported in individuals with breast cancer $[57,59]$ and colorectal cancer [60]. Participant B had rectal and breast cancer at a young age and one of her relatives had had gastric cancer. Although this variant has likely contributed to participant B's cancer risk, the risk contribution is probably small and would not lead to any special surveillance. Thus, the variant is considered of unknown significance and predictive testing cannot be offered in the family.

The variant in the HOXB13 gene, c. 251G > A, found in participant $\mathrm{A}$, is listed in ClinVar as pathogenic/likely pathogenic/risk factor (variation ID 128031, 24-03-2021) [19]. It is known to increase the risk for prostate cancer, with carriers having an estimated 4.5 times higher risk of prostate cancer than non-carriers $[61,62]$. It has also been suggested to increase the risk for colorectal cancer, with a significant association between the HOXB13 variant and colorectal cancer (OR 2.8) [63]. However, it does not increase the risk for breast cancer [64], and its potential role as a germline risk factor for other cancers is not proven. In Sweden, the Regional Cancer Committee advises against routinely testing for this $\mathrm{HOXB13}$ variant 
in families, since individuals not carrying the variant cannot be dismissed from clinical screening for prostate cancer, and the consequences of testing family members are not yet known [65]. The NCCN does not specify management of $H O X B 13$ pathogenic variant carriers but acknowledges it as a variant associated to an increased risk for prostate cancer [66]. The Philadelphia Prostate Cancer Consensus has proposed that all male $H O X B 13$ pathogenic variant carriers are offered surveillance with PSA tests from not later than 40 years of age [67]. Participant A is a woman, and no clinical action will be taken upon the finding. Her father had prostate cancer at an age of 78 and it is reasonable to believe the variant could have contributed to this.

In participant $\mathrm{B}$, a missense variant in the gene $K L L N$ was also present. Hypermethylation of the KLLN promoter is potentially causative for Cowden syndrome, which includes an increased risk for breast and colorectal cancer [68]. Participant B had indeed breast and rectal cancer, in addition to teratoma. The missense variant found in this study has not been reported before and is of unknown significance as of today.

In addition to the above, three individuals were found to be healthy carriers of pathogenic heterozygous variants that cause autosomal recessive disease (Table 2). None of these are clinically actionable in the heterozygous state and would therefore not be reported in Sweden.

Participant $\mathrm{H}$ had ongoing chronic lymphocytic leukaemia at the time of the study, and DNA isolated from the blood sample could therefore contain leukemic cells (around the time of DNA isolation from peripheral blood the leukocyte particle concentration was $20 \times 10^{9}$ / L). Several of the detected variants were below our cutoff variant allele fraction of $30 \%$, and were therefore excluded. Among the variants in Table 2, two variants in the RNF6 gene had variant allele fractions of 40 and $32 \%$, respectively, and are likely also somatic. The RNF6 gene has no known connection to inherited cancer [40].

No other likely pathogenic variants were detected. We have not analysed more common genetic risk factors. Also, we cannot rule out environmental factors contributing to the participant's cancers. Chemotherapy, hormone therapy, smoking and exposure to UV light are all known cancerogenic agents that may explain part of the clinical phenotype, but are unlikely to be responsible for the full clinical picture of the participants in this study. For example, participant D had received Tamoxifen, an anti-oestrogen treatment for her breast cancer. Tamoxifen treatment increases the risk of endometrial cancer, especially after longer use ( $>2$ years) [69]. Patient D developed endometrial cancer within 1 year after diagnosis of her breast cancer, and it is difficult to know if it was induced by the Tamoxifen or not. Of note, she had two additional primary tumours (malignant melanoma at 59 years and pancreatic carcinoma at 65 years) which cannot be attributed to Tamoxifen.

There are few studies analysing individuals with three multiple primaries. Whitworth et al. recently performed whole-genome sequencing in a cohort of 460 individuals with two or three primary tumours [12], of whom 182 had at least three multiple primaries (personal communication). Of those, 31 (17\%) harboured pathogenic or likely pathogenic variants. 30 of the 31 individuals with pathogenic or likely pathogenic variants had their first cancer diagnosed before the age of 60 . No information on family history was provided. Six individuals with breast cancer harboured risk factor variants in the $A T M$ or CHEK2 genes, and if they had close relatives with breast cancer, they would have been offered clinical genetic testing according to guidelines. Of interest, two of the detected variants were in genes rarely analysed in cancer syndromes (PTEN and NTHL1), which may easily be missed using smaller clinical gene panels. This suggests that in cases with three or more primary tumours, a broad cancer gene panel might be recommended. Once genetic testing is expanded beyond clinical criteria, there is always a risk of additional findings. Indeed, at least three of the findings by Whitworth et al. in patients with three or more primary tumours (in the genes BRCA2 and MSH2) may be classified as secondary, as they were found in individuals who had not had breast/ovarian or colorectal/endometrial cancer. Although European guidelines recommend using targeted panels to avoid incidental findings [70], several countries, including the UK [71] and France [72], have started to search for pathogenic variants in defined cancer genes after informed consent in all patients undergoing WES/WGS. In Sweden, informed consent for incidental findings is collected as part of research projects such as this one. Incidental findings in the clinical laboratory are reported after ethical review, but we do not actively search for causative variants in genes that are not known causes of the patient's clinical syndrome.

Our study is not large enough to give any significant results but it supports the data from Whitworth et al. [12]: the genetic cause in patients who fulfil clinical criteria for specific cancer syndromes may be missed by conventional testing. This is either because the patient has a genetic variant which is difficult to identify (such as the MEN1 or $M L H 1$ variant in our study), or because the syndrome is not recognised by the clinicians and therefore not tested for. One example is the NTHL1 variant in an individual with meningioma and colorectal cancer in the Whitworth study [12]. Another is a patient with a pathogenic variant in PTEN discovered by WES in a young-onset colorectal cancer cohort. Cowden 
syndrome could be confirmed clinically when the medical history was re-examined [73]. A simple re-analysis of clinical WGS/WES data would have been sufficient to diagnose $2 / 10$ individuals (20\%) with pathogenic variants in our study. Potentially, though, we could have missed variants in rare genes that are not included in the clinical panel and that cause syndromes clinicians do not recognise.

In $8 / 10(80 \%)$ of the individuals in this study, no monogenic cause of cancer was found. Four participants did not fulfil clinical criteria for any specific cancer syndrome. Multiple tumours in patients who do not fulfil testing criteria for hereditary cancer syndromes are most likely multifactorial. There might be genetic predisposition of lower penetrance in the individuals in the cohort, since we found possibly disease-related variants in cancer driver genes. WGS analysis for research purposes will probably detect interesting risk factors, including risk variants in genes such at CHEK2 and ATM, but the clinical use of WGS in these cases is still debatable. WGS/WES also has technical limitations when it comes to analysis of genes with pseudogenes and nucleotide repeat regions. Although WGS can in theory detect all disease-causing variants, some types of pathogenic variants will be missed. This may be due to lack of information on normal variation of for instance noncoding variants, or due to difficulties in detecting complex variants, such as Alu repeat insertions, using conventional bioinformatics.

In summary, we have performed WGS/WES in ten individuals with three or more primary tumours, with previously normal results from clinical genetic testing. One of the participants, with clinical Lynch-like syndrome, harboured a synonymous variant in the MLH1 gene, causing constitutional low level hypermethylation, which was considered likely pathogenic. Another participant had a clinical diagnosis of MEN1, and this study could identify a likely pathogenic cryptic splice variant in the MEN1 gene. No clinically actionable variants were detected in the other eight participants. As rare hereditary cancer syndromes are difficult to identify in routine clinical practice, WGS/WES may provide additional benefit by including a wider gene list and also by potentially being able to detect rare structural variants that may cause cancer. We therefore recommend that individuals with three primary tumours, and with their first cancer diagnosed before 60 years of age, are referred to a clinical genetics department for an evaluation. From our findings, we conclude that if a patient fulfils clinical criteria for a specific cancer syndrome, a re-evaluation of the genetic variants in genes with known association to that syndrome might be warranted after a few years.

\section{Supplementary Information}

The online version contains supplementary material available at https://doi. org/10.1186/s13053-021-00203-z.

Additional file 1: Supplementary Table S1. Sequencing coverage and reads information for each sample.

Additional file 2: Supplementary Table S2A. Gene lists; Hereditary cancer and Cancer driver. Supplementary Table S2B. Gene list; Scout HPO Neoplasia. Supplementary Table S2C. Gene list; Scout curated Clinical Genetics.

Additional file 3: Supplementary Table S3. Genes targeted in extra analysis for each participant.

Additional file 4: Supplementary Fig. S1. Schematics of the workflow for variant filtration.

\section{Acknowledgements}

We are thankful to all participants in this study, for their valuable contribution and for showing interest in the research. We would also like to thank Isabel Neira for isolating DNA from the blood samples, and Mikaela Bodell Davoody for cDNA sequencing.

\section{Authors' contributions}

ET, DN and KW contributed to the study conception and design. Material preparation, data collection and analysis were performed by all authors. The first draft of the manuscript was written by KW and all authors commented on previous versions of the manuscript. All authors read and approved the final manuscript.

\section{Funding}

Karin Wallander and Emma Tham are supported by grants from Region Stockholm (combined clinical residency and PhD training program: K28912016 and ALF-project: grant number 581046 and 500306). The computations were enabled by resources in project sens 2017620 provided by the Swedish National Infrastructure for Computing (SNIC) at UPPMAX, partially funded by the Swedish Research Council through grant agreement no. 2018-05973. Open Access funding provided by Karolinska Institute.

\section{Availability of data and materials}

The relevant datasets generated during and analysed during the current study are available from the corresponding author on reasonable request. All computational analysis can be reproduced with freely available, open source code tools: MIP (https://github.com/Clinical-Genomics/MIP) and Scout (https://github.com/Clinical-Genomics/scout)

\section{Declarations}

Ethics approval and consent to participate

All procedures performed in studies involving human participants were in accordance with the ethical standards of the institutional and national research committee and with the 1964 Helsinki Declaration and its later amendments. This study was approved by the Stockholm Regional Ethics Review Board (identification number 2012/2106-31/4). All individuals gave their written informed consent to participate in the study.

\section{Consent for publication}

All individuals gave their written informed consent to publish their data.

\section{Competing interests}

The authors claim no conflict of interest.

Received: 20 June 2021 Accepted: 12 October 2021

Published online: 28 October 2021

\section{References}

1. WHO (World Health Organisation) disease burden and mortality estimates Internet page. Available at [https://www.who.int/]. Accessed 17 Sept 2020.

2. Cancerfonden, Statistik om cancer (The Swedish Cancer Society, statistics on cancer incidence). Internet page. Available at [https://www.cancerfonden.se/ om-cancer/statistik]. Accessed 17 Sept 2020. 
3. Dong C, Hemminki K. Second primary neoplasms in 633,964 cancer patients in Sweden, 1958-1996. Int J Cancer. 2001;93(2):155-61. https://doi.org/10.1 002/ijc. 1317

4. Dickie L, Johnson C, Adams S, Negoita S. Solid Tumor Rules. Rockville: National Cancer Institute; 2019.

5. International rules for multiple primary cancers (ICD-O Third edition), IARC (International Agency for Research on Cancer). Internet page. Available at [http://www.iacr.com.fr/index.php?option=com_content\&view=article\&id= 105\&ltemid=472]. Accessed 17 Sept 2020.

6. Bittorf B, Kessler H, Merkel S, Bruckl W, Wein A, Ballhausen WG, et al. Multiple primary malignancies: an epidemiological and pedigree analysis of 57 patients with at least three tumours. Eur J Surg Oncol. 2001;27(3):302-13. https://doi.org/10.1053/ejso.2001.1112.

7. Salem A, Abu-Hijlih R, Abdelrahman F, Turfa R, Amarin R, Farah N, et al. Multiple primary malignancies: analysis of 23 patients with at least three tumors. J Gastrointest Cancer. 2012;43(3):437-43. https://doi.org/10.1007/s12 029-011-9296-7.

8. Statistics department, The Swedish Cancer Register, Swedish National Board of Health and Welfare. 2020.

9. Cybulski C, Nazarali S, Narod SA. Multiple primary cancers as a guide to heritability. Int J Cancer. 2014;135(8):1756-63. https://doi.org/10.1002/ijc.28988.

10. Naslund-Koch C, Nordestgaard BG, Bojesen SE. Increased Risk for Other Cancers in Addition to Breast Cancer for CHEK2(star)1100delC Heterozygotes Estimated From the Copenhagen General Population Study. J Clin Oncol. 2016;34(11):1208-+.

11. Xiang HP, Geng XP, Ge WW, Li H. Meta-analysis of CHEK2 1100delC variant and colorectal cancer susceptibility. Eur J Cancer. 2011;47(17):2546-51. https://doi.org/10.1016/j.ejca.2011.03.025.

12. Whitworth J, Smith PS, Martin JE, West H, Luchetti A, Rodger F, et al. Comprehensive Cancer-predisposition gene testing in an adult multiple primary tumor series shows a broad range of deleterious variants and atypical tumor phenotypes. Am J Hum Genet. 2018;103(1):3-18. https://doi. org/10.1016/j.ajhg.2018.04.013.

13. Stranneheim $H$, Lagerstedt-Robinson K, Magnusson M, Kvarnung M, Nilsson $D$, Lesko $N$, et al. Integration of whole genome sequencing into a healthcare setting: high diagnostic rates across multiple clinical entities in 3219 rare disease patients. Genome Med. 2021;13(1):40. https://doi.org/10.11 86/s13073-021-00855-5.

14. Lindstrand A, Eisfeldt J, Pettersson M, Carvalho CMB, Kvarnung M, Grigelioniene G, et al. From cytogenetics to cytogenomics: whole-genome sequencing as a first-line test comprehensively captures the diverse spectrum of disease-causing genetic variation underlying intellectual disability. Genome Med. 2019;11(1). https://doi.org/10.1186/s13073-0190675-1.

15. Online Mendelian Inheritance in Man, OMIM ๑ Johns Hopkins University, Baltimore, MD. Internet page. MIM Number: 613733 (MEN1), date last edited: 10-31-2012. Available at [https://www.omim.org/entry/613733?sea rch=men1\&highlight=men1]. Accessed 16 June 2021

16. Online Mendelian Inheritance in Man, OMIM ๑. Johns Hopkins University, Baltimore, MD. Internet page. MIM Number: 120436 (MLH1), date last edited: 01-20-2021. Available at [https://www.omim.org/entry/120436?sea $r c h=m \mid h 1$ \&highlight=mlh1]. Accessed 16 June 2021.

17. Vogelstein B, Papadopoulos N, Velculescu VE, Zhou SB, Diaz LA, Kinzler KW. Cancer genome landscapes. Science. 2013;339(6127):1546-58. https://doi. org/10.1126/science.1235122.

18. Karczewski KJ, Weisburd B, Thomas B, Solomonson M, Ruderfer DM, Kavanagh D, et al. The ExAC browser: displaying reference data information from over 60000 exomes. Nucleic Acids Res. 2017:45(D1):D840-D5. https:// doi.org/10.1093/nar/gkw971.

19. Landrum MJ, Lee JM, Benson M, Brown GR, Chao C, Chitipiralla S, et al. ClinVar: improving access to variant interpretations and supporting evidence. Nucleic Acids Res. 2018;46(D1):D1062-D7. https://doi.org/10.1093/ nar/gkx1153.

20. Alamut Visual, Splicing prediction module. Internet page. Available at [https://www.interactive-biosoftware.com/doc/alamut-visual/2.14/splicing. html]. Accessed 4 Dec 2020.

21. Mathe E, Olivier M, Kato S, Ishioka C, Hainaut P, Tavtigian SV. Computational approaches for predicting the biological effect of p53 missense mutations: a comparison of three sequence analysis based methods. Nucleic Acids Res. 2006;34(5):1317-25. https://doi.org/10.1093/ nar/gkj518.
22. Tavtigian SV, Deffenbaugh AM, Yin L, Judkins T, Scholl T, Samollow PB, et al. Comprehensive statistical study of 452 BRCA1 missense substitutions with classification of eight recurrent substitutions as neutral. J Med Genet. 2006; 43(4):295-305. https://doi.org/10.1136/jmg.2005.033878.

23. Kumar P, Henikoff S, Ng PC. Predicting the effects of coding nonsynonymous variants on protein function using the SIFT algorithm. Nat Protoc. 2009;4(7):1073-82. https://doi.org/10.1038/nprot.2009.86.

24. Adzhubei IA, Schmidt S, Peshkin L, Ramensky VE, Gerasimova A, Bork P, et al. A method and server for predicting damaging missense mutations. Nat Methods. 2010;7(4):248-9. https://doi.org/10.1038/ nmeth0410-248.

25. Schwarz JM, Rodelsperger C, Schuelke M, Seelow D. MutationTaster evaluates disease-causing potential of sequence alterations. Nat Methods. 2010;7(8):575-6. https://doi.org/10.1038/nmeth0810-575.

26. Gao JJ, Aksoy BA, Dogrusoz U, Dresdner G, Gross B, Sumer SO, et al. Integrative Analysis of Complex Cancer Genomics and Clinical Profiles Using the cBioPortal. Sci Signaling. 2013;6(269). https://doi.org/10.1126/scisignal.2 004088.

27. Richards S, Aziz N, Bale S, Bick D, Das S, Gastier-Foster J, et al. Standards and guidelines for the interpretation of sequence variants: a joint consensus recommendation of the American College of Medical Genetics and Genomics and the Association for Molecular Pathology. Genet Med. 2015; 17(5):405-24. https://doi.org/10.1038/gim.2015.30.

28. ClinGen, Clinical Genome Resource, Sequence Variant Interpretation. Internet page. Available at [https://clinicalgenome.org/working-groups/ sequence-variant-interpretation/]. Accessed 12 Sept 2021.

29. Scout, Clinical genomics. Internet page. Available at [http://www.clinica Igenomics.se/scout/]. Accessed 14 Sept 2020.

30. Rentzsch P, Witten D, Cooper GM, Shendure J, Kircher M. CADD: predicting the deleteriousness of variants throughout the human genome. Nucleic Acids Res. 2019;47(D1):D886-d94. https://doi.org/10.1093/nar/gky1016.

31. Blueprint genetics, Genetic testing for hereditary cancer. Internet page. Available at [https://blueprintgenetics.com/tests/panels/hereditary-cancer/]. Accessed 11 Feb 2021

32. Rausch T, Zichner T, Schlattl A, Stutz AM, Benes V, Korbel JO. DELLY: structural variant discovery by integrated paired-end and split-read analysis. Bioinformatics. 2012;28(18):1333-19. https://doi.org/10.1093/bioinformatics/bts378.

33. Chen XY, Schulz-Trieglaff $O$, Shaw $R$, Barnes $B$, Schlesinger F, Kallberg $M$, et al. Manta: rapid detection of structural variants and indels for germline and cancer sequencing applications. Bioinformatics. 2016;32(8):1220-2. https://doi.org/10.1093/bioinformatics/btv710.

34. Abyzov A, Urban AE, Snyder M, Gerstein M. CNVnator: an approach to discover, genotype, and characterize typical and atypical CNVs from family and population genome sequencing. Genome Res. 2011;21(6):974-84. https://doi.org/10.1101/gr.114876.110.

35. Eisfeldt J, Vezzi F, Olason P, Nilsson D, Lindstrand A. TIDDIT, an efficient and comprehensive structural variant caller for massive parallel sequencing data. F1000Res. 2017;6:664.

36. Ameur A, Dahlberg J, Olason P, Vezzi F, Karlsson R, Martin M, et al. SweGen: a whole-genome data resource of genetic variability in a cross-section of the Swedish population. Eur J Hum Genet. 2017;25(11):1253-60. https://doi. org/10.1038/ejhg.2017.130.

37. Lemos MC, Thakker RV. Multiple endocrine neoplasia type 1 (MEN1): analysis of 1336 mutations reported in the first decade following identification of the gene. Hum Mutat. 2008;29(1):22-32.

38. The Melanoma Genetics Consortium: GenoMEL, the Melanoma Genetics Consortium; 2015. Available at http://genomel.org/. Accessed 13 Feb 2021.

39. Swedish national health care guidelines for malignant melanoma (Svenska nationella vårdprogrammet för malignt melanom) 2019-12-09. Available at https://cancercentrum.se/samverkan/cancerdiagnoser/hud-och-ogon/va rdprogram/. Accessed 13 Feb 2021

40. OMIM, Online Mendelian inheritance in man. Available at https://omim.org/. Accessed 13 Feb 2021

41. Karczewski KJ, Francioli LC, Tiao G, Cummings BB, Alföldi J, Wang Q, et al. The mutational constraint spectrum quantified from variation in 141,456 humans. Nature. 2020;581(7809):434-43.

42. Tham E, Grandell U, Lindgren E, Toss G, Skogseid B, Nordenskjöld M. Clinical testing for mutations in the MEN1 gene in Sweden: a report on 200 unrelated cases. J Clin Endocrinol Metab. 2007;92(9):3389-95.

43. ClinVar, search for the MEN1 gene. Available at https://www.ncbi.n/m.nih. gov/clinvar. Accessed 15 Mar 2021. 
44. Romanet P, Odou MF, North MO, Saveanu A, Coppin L, Pasmant E, et al. Proposition of adjustments to the ACMG-AMP framework for the interpretation of MEN1 missense variants. Hum Mutat. 2019;40(6):661-74.

45. Brandi ML, Agarwal SK, Perrier ND, Lines KE, Valk GD, Thakker RV. Multiple endocrine neoplasia type 1: latest insights. Endocr Rev. 2021;42(2):133-70.

46. Backman S, Bajic D, Crona J, Hellman P, Skogseid B, Stålberg P. Whole genome sequencing of apparently mutation-negative MEN1 patients. Eur Endocrinol. 2020;182(1):35-45.

47. Ward RL, Dobbins T, Lindor NM, Rapkins RW, Hitchins MP. Identification of constitutional MLH1 epimutations and promoter variants in colorectal cancer patients from the Colon Cancer Family Registry. Genet Med. 2013; 15(1):25-35.

48. Leclerc J, Flament C, Lovecchio T, Delattre L, Ait Yahya E, Baert-Desurmont $\mathrm{S}$, et al. Diversity of genetic events associated with MLH1 promoter methylation in Lynch syndrome families with heritable constitutional epimutation. Genet Med. 2018;20(12):1589-99.

49. Hiatt SM, Amaral MD, Bowling KM, Finnila CR, Thompson ML, Gray DE, et al. Systematic reanalysis of genomic data improves quality of variant interpretation. Clin Genet. 2018;94(1):174-8.

50. Costain G, Jobling R, Walker S, Reuter MS, Snell M, Bowdin S, et al. Periodic reanalysis of whole-genome sequencing data enhances the diagnostic advantage over standard clinical genetic testing. Eur J Hum Genet. 2018; 26(5):740-4

51. Stafford JL, Dyson G, Levin NK, Chaudhry S, Rosati R, Kalpage H, et al. Reanalysis of BRCA1/2 negative high risk ovarian cancer patients reveals novel germline risk loci and insights into missing heritability. PLoS One. 2017;12(6):e0178450

52. Tung N, Domchek SM, Stadler Z, Nathanson KL, Couch F, Garber JE, et al. Counselling framework for moderate-penetrance cancer-susceptibility mutations. Nat Rev Clin Oncol. 2016;13(9):581-8.

53. Schmidt MK, Hogervorst F, Hien Rv, Cornelissen S, Broeks A, Adank MA, et al. Age- and tumor subtype-specific breast cancer risk estimates for CHEK2*1100delC carriers. J Clin Oncol. 2016;34(23):2750-60.

54. Nationellt vårdprogram för bröstcancer (Regional cancer centra, Sweden). Available at https://kunskapsbanken.cancercentrum.se/diagnoser/brostca ncer/vardprogram/. Accessed 17 Sept 2020.

55. National Comprehensive Cancer Network. Genetic/familial high-risk assessment: Breast, ovarian, and pancreatic (Version 1.2021). Available at https://www.nccn.org/professionals/physician_gls/pdf/genetics_bop.pdf. Accessed 17 Oct 2020

56. Taylor A, Brady AF, Frayling IM, Hanson H, Tischkowitz M, Turnbull C, et al. Consensus for genes to be included on cancer panel tests offered by UK genetics services: guidelines of the UK Cancer Genetics Group. J Med Genet. 2018;55(6):372-7.

57. Roeb W, Higgins J, King MC. Response to DNA damage of CHEK2 missense mutations in familial breast cancer. Hum Mol Genet. 2012;21(12):2738-44.

58. Han FF, Guo CL, Liu LH. The effect of CHEK2 variant I157T on Cancer susceptibility: evidence from a meta-analysis. DNA Cell Biol. 2013;32(6):329-35.

59. Kleibl Z, Havranek O, Novotny J, Kleiblova P, Soucek P, Pohlreich P. Analysis of CHEK2 FHA domain in Czech patients with sporadic breast cancer revealed distinct rare genetic alterations. Breast Cancer Res Treat. 2008; 112(1):159-64.

60. Kleibl Z, Havranek O, Hlavata I, Novotny J, Sevcik J, Pohlreich P, et al. The CHEK2 gene I157T mutation and other alterations in its proximity increase the risk of sporadic colorectal cancer in the Czech population. Eur J Cancer. 2009;45(4):618-24.

61. Huang H, Cai B. G84E mutation in HOXB13 is firmly associated with prostate cancer risk: a meta-analysis. Tumor Biol. 2014;35(2):1177-82.

62. Xu JF, Lange EM, Lu LY, Zheng SQL, Wang Z, Thibodeau SN, et al. HOXB13 is a susceptibility gene for prostate cancer: results from the international consortium for prostate cancer genetics (ICPCG). Hum Genet. 2013;132(1):5-14.

63. Akbari MR, Anderson LN, Buchanan DD, Clendenning M, Jenkins MA, Win AK, et al. Germline HOXB13 p.Gly84Glu mutation and risk of colorectal cancer. Cancer Epidemiol. 2013;37(4):424-7.

64. Liu J, Prager - van der Smissen WJC, Collée JM, Bolla MK, Wang Q, Michailidou K, et al. Germline HOXB13 mutations p.G84E and p.R217C do not confer an increased breast cancer risk. Sci Rep. 2020;10(1):9688.

65. Nationellt vårdprogram, Prostatacancer, version 5, 03-03-2020 (Swedish National Care Program, Prostate cancer) Available at https://www.ca ncercentrum.se/globalassets/cancerdiagnoser/prostatacancer/vardprogram/ nationellt-vardprogram-prostatacancer.pdf. Accessed 01 Oct 2020.
66. National Comprehensive Cancer Network. Prostate cancer early detection (Version 2.2020). Available at https://www.nccn.org/professionals/physician_ gls/pdf/prostate_detection.pdf. Accessed 17 Oct 2020.

67. Giri VN, Knudsen KE, Kelly WK, Abida W, Andriole GL, Bangma CH, et al. Role of genetic testing for inherited prostate cancer risk: Philadelphia prostate cancer consensus conference 2017. J Clin Oncol. 2018;36(4):414-24.

68. Nizialek EA, Mester JL, Dhiman VK, Smiraglia DJ, Eng C. KLLN epigenotypephenotype associations in Cowden syndrome. Eur J Hum Genet. 2015; 23(11):1538-43.

69. Bergman L, Beelen MLR, Gallee MPW, Hollema H, Benraadt J, van Leeuwen $\mathrm{FE}$, et al. Risk and prognosis of endometrial cancer after tamoxifen for breast cancer. Lancet. 2000;356(9233):881-7.

70. Claustres M, Kožich V, Dequeker E, Fowler B, Hehir-Kwa JY, Miller K, et al. Recommendations for reporting results of diagnostic genetic testing (biochemical, cytogenetic and molecular genetic). Eur J Hum Genet. 2014; 22(2): 160-70.

71. Ormondroyd E, Mackley MP, Blair E, Craft J, Knight JC, Taylor JC, et al. "Not pathogenic until proven otherwise": perspectives of UK clinical genomics professionals toward secondary findings in context of a genomic medicine multidisciplinary team and the 100,000 genomes project. Genet Med. 2018; 20(3):320-8. https://doi.org/10.1038/gim.2017.157.

72. Pujol P, Vande Perre P, Faivre L, Sanlaville D, Corsini C, Baertschi B, et al. Guidelines for reporting secondary findings of genome sequencing in cancer genes: the SFMPP recommendations. Eur J Hum Genet. 2018;26(12): 1732-42. https://doi.org/10.1038/s41431-018-0224-1.

73. Thutkawkorapin J, Lindblom A, Tham E. Exome sequencing in 51 early onset non-familial CRC cases. Mol Genet Genomic Med. 2019;7(5):e605. https://doi.org/10.1002/mgg3.605.

\section{Publisher's Note}

Springer Nature remains neutral with regard to jurisdictional claims in published maps and institutional affiliations.

Ready to submit your research? Choose BMC and benefit from:

- fast, convenient online submission

- thorough peer review by experienced researchers in your field

- rapid publication on acceptance

- support for research data, including large and complex data types

- gold Open Access which fosters wider collaboration and increased citations

- maximum visibility for your research: over $100 \mathrm{M}$ website views per year

At BMC, research is always in progress.

Learn more biomedcentral.com/submissions 